\title{
Comptes Rendus Chimie
}

Marc Fontecave et Dominique Grand

Corrigendum : Les scénarios énergétiques à l'épreuve du stockage des énergies intermittentes

Volume 24, issue 3 (2021), p. 415

Published online: 16 December 2021

https://doi.org/10.5802/crchim.133

(c) BY This article is licensed under the

Creative Commons Attribution 4.0 International License.

http://creativecommons.org/licenses/by/4.0/

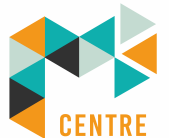

MERSENNE

Les Comptes Rendus. Chimie sont membres du

Centre Mersenne pour l'édition scientifique ouverte

www.centre-mersenne.org

e-ISSN : 1878-1543 


\title{
Corrigendum : Les scénarios énergétiques à l'épreuve du stockage des énergies intermittentes
}

\section{Corrigendum: Energy scenarios to the test of intermittent energy storage}

\author{
Marc Fontecave $^{\oplus *}, a$ et Dominique Grand ${ }^{b}$

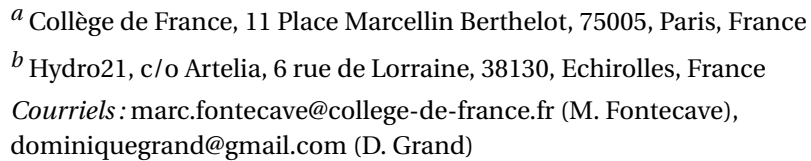

Manuscrit reçu le 20 octobre 2021, accepté le 26 octobre 2021.

Cette note apporte une correction ou un complément à l'article des Comptes Rendus. Chimie portant le titre ci-dessus. L'article concerné a été publié en ligne le 22 septembre 2021, dans le Volume 24, Numéro 2, 2021, pages 331-350, https://doi.org/10.5802/crchim. 115.

Afin de réparer une omission et d'étayer d'une référence la présentation des performances des batteries de véhicules électriques, la phrase, p. 343, milieu de $2^{\text {ème }}$ colonne :
"Aujourd'hui, on en sait plus sur la véritable durée des batteries et les nouvelles sont plutôt très bonnes.»

doit être complétée de la référence indiquée :

"Aujourd'hui, on en sait plus sur la véritable durée des batteries et les nouvelles sont plutôt très bonnes [15b].»

[15b] D. Bloch, Revue Passages, 2021, 208-209, 57-60.

Les auteurs s'excusent du désagrément.

\footnotetext{
* Auteur correspondant.
} 